THEMATIC PAPER

Reflections on research and clinical collaborations between South Asia and the UK

\author{
Athula Sumathipala, ${ }^{1}$ Abhijit Nadkarni ${ }^{2}$ and Chesmal Siriwardhana ${ }^{3}$
}

1Professor of Psychiatry, Research Institute for Primary Care and Health Sciences, Keele University, UK; Honorary Consultant, South Staffordshire and Shropshire Healthcare Foundation Trust UK; Honorary Director, Institute for Research and Development, Sritanka, enal asumathipan, Sri Lanka, email a.sumathipala@ keele.ac.uk

${ }^{2}$ Research Fellow, London School of Hygiene and Tropical don, UK; Sangath, Goa, India

${ }^{3}$ Senior Lecturer in Public Health, Faculty of Medical Science, Anglia Ruskin University, UK: Centre for Global Mental Centre for Global Mental
Health, Institute of Psychiatry, Psychology and Neuroscience, King's College London, UK; Institute for Research and Development, Sri Lanka
In this paper we describe the mutual benefits of the bi-directional flows of knowledge and skills from North-South/South-North linkages, on the basis of our personal experiences of clinical and research collaborations between the UK and South Asia.

The '10/90' gap refers to the enormous inequity in global healthcare and research: $<10 \%$ of research funds are spent on the diseases that account for $90 \%$ of the global disease burden (Global Forum for Health Research, 2000; Razzouk et al, 2010). This inequity is reflected in a publication gap (Patel \& Sumathipala, 2001; Sumathipala et al, 2004). Although the treatment gap for mental disorders in low- and middle-income countries (LMICs) is frequently highlighted (Prince et al, 2007), a recent report by the UK's Chief Medical Officer shows that the picture is not much different in high-income countries, with only $25 \%$ of those with mental health problems in the UK accessing mental health services (Chief Medical Officer, 2013).

Historically, the tendency has been to highlight the potential of the West (global North) in building the capacity in resource-poor settings of the East (global South). However, during more recent times there has been a paradigm shift in how these traditional relationships are viewed. In this paper we seek to elucidate North-South relationships by describing the mutual and interdependent benefits of the bi-directional flows of knowledge and skills from North-South/South-North linkages, using our personal experiences of clinical and research collaborations between the UK and South Asia.

Our collective experiences stem from several South Asia-UK collaborations, including:

- the sponsorship programme of the Royal College of Psychiatrists

- the Maudsley International continuing professional development (CPD) peer group

- the UK-Sri Lanka Trauma Group

- the partnership between the Institute for Research and Development (IRD) in Sri Lanka and the Institute of Psychiatry, Psychology and Neuroscience (IoPPN) in the UK

- the South Asian Hub for Advocacy, Research and Education in mental health (SHARE)
- the Programme for Improving Mental Health Care (PRIME).

Some of these programmes exclusively involve clinical training and service development, while others are research collaborations. The selected examples reflect our personal involvement and are not exhaustive.

International links and exchanges can be formal or informal, with the latter having a more individual-oriented approach. However, both types can lead to benefits. Our examples reflect links that have resulted in short- and long-term benefits for both the individuals and the institutions involved.

\section{Clinical exchanges}

The Maudsley International initiative (http://www. maudsleyinternational.com) is a formal clinical collaboration on the part of clinicians, managers and scientists, to provide consultancy, teaching and training services tailored to improve global mental health by mutual sharing of expertise among a diverse range of collaborators around the world. It includes experts at the South London and Maudsley NHS Foundation Trust and the IoPPN. This initiative has generated benefits globally, in terms of mutual learning and consolidation of expertise and a bi-directional transfer of a wealth of experience from diverse cultures and geographical locations.

The sponsorship programme led by the Royal College of Psychiatrists is designed to allow a small number of doctors to enter the UK from outside the European Union (EU) for up to 24 months, to benefit from training in the National Health Service (NHS) before returning to their home countries. Under this scheme, International Fellows are placed in training posts with NHS trusts.

\section{Research exchanges}

There are critical advantages of North-South collaborative partnerships in building research capacity (Siriwardhana et al, 2011). The collaborative research partnership between the IoPPN, King's College London, and the IRD in Sri Lanka is a classic example of a successful North-South initiative contributing to research capacity building (Siriwardhana et al, 2011). A.S. was an initiator of this programme in 1997, and C.S. is a beneficiary and a contributor to this continuing and developing partnership (Siriwardhana et al, 2011; Sumathipala et al, 2013). The IoPPN-IRD collaboration has 
led to the establishment of one of the largest twin registries in a low-income country, several globally important research studies and several capacitybuilding programmes (Siriwardhana et al, 2011; Sumathipala et al, 2013).

Sangath (a non-governmental, not-for-profit organisation) in Goa, India, and the Human Development Research Foundation (HDRF) in Pakistan are other such organisations involved as key partners representing South Asia in two major international research capacity-building consortiums: SHARE and PRIME. SHARE, funded by the National Institute of Mental Health, is a collaboration between the London School of Hygiene and Tropical Medicine and the IoPPN in the UK, and a network of research institutions and nongovernmental organisations (NGOs) across India, Afghanistan, Nepal, Bangladesh and Sri Lanka (see http://sangath.com/inside_page.php?nav_id=232). PRIME is a programme in Ethiopia, Uganda, South Africa, India and Nepal funded by the UK Department for International Development (DfID). It has a focus on health systems strengthening through working partnerships between academic researchers, NGOs and the World Health Organization (Lund et al, 2012).

Focused training courses can strengthen the research and leadership skills of mental health professionals in LMICs. The Leadership in Mental Health (LMH) course has been jointly offered by the London School of Hygiene and Tropical Medicine and Sangath for the past 6 years (see http://www.sangath.com/images/file/LMH2014_ Sept\%2012.pdf). A.N. has been involved in the 2-week course, which equips participants with research and leadership skills to develop and scale up interventions for people with mental disorders in low-resource settings, and to promote the human rights of people affected by mental disorders. Since its inception, the LMH has hosted more than 200 participants from around the world, including mental health practitioners, researchers, policy makers, persons affected by mental health problems and advocates from countries as diverse as Nepal, Norway, Mexico, the USA, Australia, Nigeria, Jordan, Canada and Afghanistan.

C.S. and A.S. have led the organisation of a 'Qualitative methods for health research' programme at IRD since 2011, aiming to develop capacity to conduct qualitative health research in Sri Lanka and the wider South Asian region. We have hosted 80 or so participants from Sri Lanka and other South Asian countries. This is a partnership between the IoPPN, King's College London, the Institute of Public Health, the University of Cambridge and Anglia Ruskin University in the UK and SHARE.

\section{The balance of benefits}

At a glance, the work described above may appear to benefit the Southern partners. However, is this an accurate assessment? In this section, we explore the balance of benefits in North-South partnerships, challenges in establishing productive exchanges and the role of reverse innovation.

It is beyond doubt that overseas recruitment schemes are golden opportunities for LMIC doctors to get work experience in the world's best healthcare systems. However, some perceive it as trying to fill jobs in specialties where there is a recruitment crunch. One of the major challenges faced by the LMICs is the 'brain drain' and experience suggests that very few LMIC doctors return to their home countries after the training scheme has ended, thus increasing local shortages of healthcare professionals (Patel, 2003). Patel (2003) has been highly critical of one NHS scheme, now discontinued, which effectively poached highly experienced specialists in a number of fields, including psychiatry. He accuses the NHS of opportunism, where it should have been providing opportunities. While we agree that there certainly is a case to answer, as the benefits of clinical exchanges tend to be unfairly weighted towards the North, does the blame for robbing the best brains from the South lie only with the North?

As stated by Patel, when he finished training in psychiatry in the UK he found that very few routes were available to facilitate his return to India to practise (Patel \& Araya, 1992). The difficulties that doctors face when they attempt to return home have not been widely discussed. As many of those trained in the global North would testify, there is resistance from their colleagues in their home countries to integrating those returning. A.S. has very poignant personal experience related to this issue. A.N., too, has first-hand experience of the challenges and advantages of returning to his home country after training in the UK. The continued links with academic institutions in the UK allow him to access CPD and also to contribute to teaching activities in the UK. Such an arrangement does not come without its share of challenges but, if one is able to go through the initial barriers, a vastly productive work environment can be created.

The research collaborations described above help the LMICs by strengthening individual and institutional capacity. C.S. has benefited through the IoPPN-IRD research partnership, gaining fellowship funding from the Wellcome Trust as a result and completing a masters degree and a $\mathrm{PhD}$ at the IoPPN while conducting research in Sri Lanka. However, there have been challenges in being based at two locations, dealing with systems built on different hierarchical attitudes, and resistance encountered when promoting a globally oriented research culture.

Research collaborations are reciprocally advantageous, as the knowledge translation and exchange allow evidence generation in the LMICs which can enormously contribute to knowledge generation in the health systems of high-income countries. For example, increasing treatment coverage through delivery of mental healthcare using non-specialist health workers used in LMICs would be useful if replicated in high-income countries, especially in recent times of economic difficulties. 
In our experience, international clinical and research exchange can make a significant contribution to mutual learning and the consolidation of expertise. It represents a bi-directional transfer of the knowledge and experience between the North and the South.

\section{References}

Chief Medical Officer (2013) Annual Report of the Chief Medical Officer 2013: Public Mental Health Priorities: Investing in the Evidence. Available at https:/www.gov.uk/government/ uploads/system/uploads/attachment_data/file/351629/Annual_ report_2013_1.pdf (accessed November 2014).

Global Forum for Health Research (2000) The 10/90 Report on Health Research. GFHR.

Lund, C., Tomlinson, M., De Silva, M., et al (2012) PRIME: a programme to reduce the treatment gap for mental disorders in five low- and middle-income countries. PLoS Medicine, 9(12), e1001359.

Patel, V. (2003) Recruiting doctors from poor countries: the great brain robbery? BMJ, 327, 926-928.
Patel, V. \& Araya, R. (1992) Trained overseas, unable to return home: plight of doctors from developing countries. Lancet, 339, 110-111.

Patel, V. \& Sumathipala, A. (2001) International representation in psychiatric literature. Survey of six leading journals. British Journal of Psychiatry, 178, 406-409.

Prince, M., Patel, V., Saxena, S., et al (2007) No health without mental health. Lancet, 370, 859-877.

Razzouk, D., Sharan, P., Gallo, C., et al (2010) Scarcity and inequity of mental health research resources in low- and-middle income countries: a global survey. Health Policy, 94, 211-220.

Siriwardhana, C., Sumathipala, A., Siribaddana, S., et al (2011) Reducing the scarcity in mental health research from low and middle income countries: a success story from Sri Lanka. International Review of Psychiatry, 23, 77-83.

Sumathipala, A., Siribaddana, A. \& Patel, V. (2004) Underrepresentation of developing countries in the research literature: ethical issues arising from a survey of five leading medical journals. BMC Medical Ethics, 5:5, doi:10.1186/1472-6939-5-5.

Sumathipala, A., Siribaddana, S., Hotopf, M., et al (2013) The Sr Lankan Twin Registry: 2012 update. Twin Research and Human Genetics, 16, 307-312.

\section{THEMATIC} PAPER

\title{
Diaspora and peer support working: benefits of and challenges for the Butabika-East London Link
}

\author{
Dave Baillie, ${ }^{1}$ Mariam Aligawesa, ${ }^{2}$ Harriet Birabwa-Oketcho, ${ }^{3}$ Cerdic Hall, ${ }^{4}$ \\ David Kyaligonza, ${ }^{5}$ Richard Mpango, ${ }^{6}$ Moses Mulimira ${ }^{7}$ and Jed Boardman ${ }^{8}$
}

'Consultant Psychiatrist, and Chair, Butabika-East London Link, East London NHS Foundation Trust, London, UK, email davewhbaillie@gmail.com

${ }^{2}$ Charge Nurse, Co-Chair, Butabika East-London Link, and Co-chair, Ugandan Diaspora Health Foundation, South London and Maudsley NHS Foundation Trust, London, UK

${ }^{3}$ Consultant Psychiatrist, Butabika Hospital, Kampala, Uganda

${ }^{4}$ Nurse Consultant, Camden and Islington NHS Foundation Trust, London, UK

${ }^{5}$ Director of Nursing, Butabika Hospital, Kampala, Uganda ${ }^{6}$ Head Occupational Therapist, Butabika Hospital, Kampala,

Uganda

${ }^{7}$ Life Skills and Recovery Mental Health Worker, and Co-chair Butabika-East London Link, East Bust London NHS London, UK

${ }^{8}$ Consultant and Senior Lecturer in Social Psychiatry, South London and Maudsley NHS Foundation Trust, London, UK
The International Health Partnership ('the Link') between the East London NHS Foundation Trust and Butabika Hospital in Uganda was set up in 2005. It has facilitated staff exchanges and set up many workstreams (e.g. in child and adolescent psychiatry, nursing and psychology) and projects (e.g. a peer support worker project and a violence reduction programme). The Link has been collaborative and mutually beneficial. The authors describe benefits and challenges at individual and organisational levels. Notably, the Link has achieved a commitment to service user involvement and an increasingly central involvement of the Ugandan diaspora working in mental health in the UK.

International health partnerships (or Links) are formalised voluntary partnerships between UK health institutions and counterpart health institutions in low-income countries, intended for mutual benefit, through sharing of learning, experience and capacity building. Over 25 years, the Tropical Health and Education Trust (THET) has supported and built a partnership approach that aims to harness knowledge and technical expertise to strengthen health systems through the training of healthcare workers in low-income settings (THET, 2009). It is involved in the allocation of Department for International Development (DfID) funds to Links. Links are advocated as a vehicle for international development (Crisp, 2007) with potential benefits to individual health workers (Longstaff, 2012) as well as UK institutions (All Parliamentary Party Group on Global Health, 2013, 2014; Forrington et al, 2014).

The Butabika-East London Link (Baillie $e t$ al, 2009) was set up in 2005 between interested staff at an English mental health trust (East London NHS Foundation Trust, ELFT) and Butabika Hospital, the national referral and teaching psychiatric hospital in Uganda. Since then, 40 Ugandan staff have come to London for training and over 70 UK staff have visited Uganda, collaborating on a series of work-streams and projects (see Box 1). Exchange staff have included psychiatrists, nurses, occupational therapists, social workers, psychologists, pharmacists, service users and administrative staff. 